\title{
Viscous energy dissipation of kinetic energy of particles comminuted by high-rate shearing in projectile penetration, with potential ramification to gas shale
}

\author{
Yewang Su · Zdeněk P. Bažant • Youxuan Zhao • \\ Marco Salviato · Kedar Kirane
}

Received: 2 January 2015 / Accepted: 11 April 2015 / Published online: 29 April 2015

(C) Springer Science+Business Media Dordrecht 2015

\begin{abstract}
While dynamic comminution is of interest to many processes and situations, this work is focused on the projectile impact onto concrete walls, in which predictions have been hampered by the problem of the so-called 'dynamic overstress'. Recently, in analogy with turbulence, Bažant and Caner modeled the overstress as an additional viscous stress generated by apparent viscosity that accounts for the energy dissipation due to kinetic comminution of concrete into small particles at very high shear strain rate. Their viscosity estimation, however, was approximate since it did not satisfy the energy balance exactly. Here their model is extended and refined by ensuring that the drop of local kinetic energy of high shear strain rate of form-
\end{abstract}

Yewang Su was on leave from State Key Laboratory of Nonlinear Mechanics, Institute of Mechanics, Chinese Academy of Sciences, Beijing 100190, China.

Youxuan Zhao was on leave from School of Civil Engineering, Southwest Jiaotong University, Chengdu, China.

Y. Su $\cdot$ Y. Zhao $\cdot$ M. Salviato

Department of Civil and Environmental Engineering,

Northwestern University, Evanston, IL 60208, USA

\section{Z. P. Bažant ( $\varangle)$}

McCormick Institute Professor and W.P. Murphy Professor

of Civil and Mechanical Engineering and Materials

Science, Northwestern University, Tech Rm A135,

2145 Sheridan Rd., Evanston, IL 60208, USA

e-mail: z-bazant@northwestern.edu

K. Kirane

Department of Mechanical Engineering, Northwestern

University, Evanston, IL 60208, USA ing particles must be exactly equal to the energy dissipated by interface fracture of these particles. The basic hypothesis is that the interface fracture occurs instantly, as soon as the energy balance is satisfied. Like in the preceding work, this additional apparent viscosity is a power function of the rate of the deviatoric strain invariant. But here the power exponent is different, equal to $-7 / 3$, and the apparent viscosity is found to be proportional also to the time derivative of the rate of that invariant, i.e., to the second derivative of the shear strain. It is assumed that the interface fracture that comminutes the material into small particles occurs instantly, as soon as the local kinetic energy of shear strain rate in the forming particles becomes equal to the energy required to form interface fractures. The post-comminution behavior, including subsequent further comminution and clustering into bigger particle groups to release the kinetic energy that is being dissipated by inter-group friction, is discussed and modeled. The present formulation makes it possible to eliminate the artificial damping of all types, which is normally embedded in commercial finite element codes but is not predictive since it is not justified physically. With the aforementioned improvements, and after implementation into the new microplane model M7 for fracturing damage in concrete (which includes the quasi-static rate effects), the finite element predictions give superior agreement with the measured exit velocities of steel projectiles penetrating concrete walls of different thicknesses and with the measured depths of penetration into concrete blocks by projectiles of different veloci- 
ties. Finally it is pointed out that the theory presented may also be used to predict proximate fragmentation and permeability enhancement of gas shale by powerful electric pulsed-arc explosions in the borehole.

Keywords Comminution - Fragmentation - High strain rate - Impact - Dynamic fracture - Fracture mechanics $\cdot$ Concrete $\cdot$ Gas shale

\section{Introduction}

Dynamic comminution (i.e., fragmentation, crushing and pulverization), due to high shear strain rate occurs in many situations and processes, ranging from projectile or meteorite impact on rocks, concrete, composites, metals and ceramics (Deshpande and Evans 2008; Doyoyo 2002; Ferri et al. 2010; Mescall and Weiss 1984; Wei et al. 2009), to fracturing of gas or oil shale by chemical explosions or electro-hydraulic pulsed arc in the horizontal borehole (Hemmert et al. 2010; Maurel et al. 2010). There exists a vast literature on the subject (Adley et al. 2012; Cadoni et al. 2001; Camacho and Ortiz 1996; Deshpande and Evans 2008; Doyoyo 2002; Ferri et al. 2010; Forquin et al. 2008; Frank et al. 2012; Gailly and Espinosa 2002; Gatuingt and Pijaudier-Cabot 2002; Grady 1982; Grady 1998; Grady and Kipp 1979; Grady and Kipp 1995; Grady 1990; Kožar and Ožbolt 2010; Mescall and Weiss 1984; Mott 1947; Ožbolt et al. 2011; Shih et al. 1998; Wei et al. 2009) and many aspects are well understood, including the micro-mechanics of dynamic propagation and branching of individual cracks (Freund 1990).

The material comminution represents an additional process of energy dissipation. The multiscale transition needed to translate the aforementioned models for individual cracks into global energy dissipation of vast number of cracks and develop a general constitutive law usable in large finite element programs for impact seems hardly feasible. The most important constitutive phenomenon, which has eluded understanding for decades, is the dynamic overstress. It consists of an apparent increase of strength that begins at the shear strain rate of about $1 / \mathrm{s}$ and becomes enormous at shear strain rates $>10^{4} / \mathrm{s}$. Some authors were able to simulate the dynamic overstress under impact by either introducing into the finite element model various kinds of suitably strong artificial damping, or by arbitrarily increasing the strength or yield limits of the material so as to fit some impact test data (Adley et al. 2012). However, such ad hoc modifications deprive the constitutive law of its experimental foundation in the fitting of laboratory tests of uni-, bi- and tri-axial behavior under diverse loading paths. Consequently, such modifications cease to be predictive in new situations, although they might generate realistically looking videos. A sound way to model the dynamic overstress must be a physically justified refinement, rather than ad hoc modification, of the damage constitutive model.

Such refinement has recently been attempted with considerable success in the work of Bažant and Caner (2013), Bažant and Caner (2014) and Caner and Bažant (2014). The underlying constitutive model was the microplane model M7 for concrete, which includes only the quasi-static rate effects. M7 was combined with a new theory in which the particle-producing interface fracture of concrete was considered to be driven by the release of the kinetic energy of the local shear strain field, rather than by the release of strain energy as in the classical fracture mechanics. This was justified by the fact that the former energy release can be several magnitudes larger than the latter. The energy dissipated by comminution into particles was modeled by additional viscosity based on the energy dissipated by the comminution (while all the types of artificial damping usually embedded in commercial dynamic finite element programs were removed). The model agreed reasonably well with published test data (Cargile 1999) on the exit velocities of projectiles penetrating concrete walls of different thicknesses. Nevertheless the model contained one crude oversimplification, which is removed in the present study.

The oversimplification in the previous work (Bažant and Caner 2013; Bažant and Caner 2014; Caner and Bažant 2014) was due to calculating the additional shear viscosity so as to yield a dissipation rate proportional to the deviatoric stress invariant of the shear stress required to drive interface fracture. What prevented this simplification from being unrealistic was that this invariant had the correct physical dimension, the energy per unit volume. But the value of the proportionality constant had to be left to data fitting, and the relationship of that invariant to energy dissipation and viscosity was only approximate, even though satisfactory fits of the exit velocities of penetrating projectiles were obtained. Here it is shown how to calculate the additional viscosity in a fully consistent manner, to obtain more accurate results. 
The previous work also introduced the hypothesis that the interface fracture that produces the comminuted particles occurs instantaneously. This point is debatable. On one hand, the fracture doubtless begins at the same instant at many forming particles, rather than propagating from one particle to the next. This supports the hypothesis of instantaneity. On the other hand, the fracture certainly propagates along each particle interface, and the propagation is surely rate-dependent (this alternative is explored in a parallel paper Bažant and Su 2015). The present work develops the former alternative, i.e., the instantaneous fracture formation at all particle interfaces while the balance of fracturing and viscous energy dissipation is strictly maintained.

\section{Drop of kinetic energy density caused by comminution: a review}

Let $\epsilon_{D i j}$ denote the deviatoric strain tensor and the superior dot, ', the derivatives with respect to time $t$. Consider an idealized dynamic fracture process in which the solid is comminuted to identical prismatic particles of length $h$ and hexagonal cross section of side $h / 2$, as shown in Fig. 1 . The kinetic energy of effective deviatoric strain rate $\dot{\epsilon}_{D}=\sqrt{\dot{\epsilon}_{D i j} \dot{\epsilon}_{D i j} / 2}$ becomes, at a certain moment, high enough to create sudden fracture at particle interfaces.

Analyzing the kinematics and comparing the kinetic energy of particles before (Fig. 1b) and after (Fig. 1c) the comminution, Bažant and Caner (2014) showed that, for a motion in the plane of maximum shear strain, the local kinetic energy of the particles that are about to form, per unit volume of material, $\Delta \mathcal{K}$, is additive to, and separable from, the kinetic energy of the global motion. The global kinetic energy corresponds to the movement of the centers of the particles whose formation is imminent. Thus the drop of kinetic energy per unit volume is found to be

Fig. 1 Schematic illustration of material comminution into prismatic hexagonal particles: a undeformed regime; $\mathbf{b}$ sheared regime; $\mathbf{c}$ comminuted regime
$-\Delta \mathcal{K}=c_{k} \rho h^{2} \dot{\epsilon}_{D}^{2}$

where $c_{k}=I_{p} /\left(2 h V_{p}\right), \rho=$ mass density, $V_{p}=$ $3 \sqrt{3} h^{3} / 8$ and $I_{p}=5 \sqrt{3} h^{4} / 128=$ volume and polar moment of inertia of each hexagonal prism about its axis, respectively.

In reality, the size of comminuted particles is never uniform but varies randomly within a certain range, $s \in(h, H)$ where $h, H=$ minimum and maximum sizes, and $s=$ variable particle size. Schuhmann's empirical power law (Charles 1957; Ouchterlony 2005; Schuhmann (1940)) is adopted to describe the cumulative distribution (Fig. 2). It gives the volume fraction of particles of sizes between $h$ to $s$ :

$F(s)=\frac{s^{k}-h^{k}}{H^{k}-h^{k}}, \quad s \in(h, H), F(s) \in(0,1)$

where $(k \approx 0.5)=$ empirical constant (Bažant and Caner 2014).

We now introduce the simplifying hypothesis that the kinetic energy drop for variable particle size can be obtained by summing the energy losses for all infinitesimal intervals $(s, s+\mathrm{d} s)$ calculated for each interval as if the particle size and shape were uniform. Integration furnishes the kinetic energy drop for particles of all sizes per unit volume (Bažant and Caner 2014):

$-\Delta \mathcal{K}=\int_{s=h}^{H} c_{k} \rho s^{2} \dot{\epsilon}_{D}^{2} \mathrm{~d} F(s)=C_{k} \rho h^{2} \dot{\epsilon}_{D}^{2}$

where $C_{k}=\frac{k}{k+2} \frac{(H / h)^{k+2}-1}{(H / h)^{k}-1} c_{k}$

$C_{k}=$ dimensionless constant. For $H / h=10$ and $k=$ $0.5, C_{k}=1.519$.

The interface area of uniform particle of size $s$ per unit volume of material is $c_{s} / s$ where $c_{s}=$ dimensionless constant. For particle size distributed according to Eq. (2), the particle interface area per unit volume of material is obtained as (Bažant and Caner 2014):
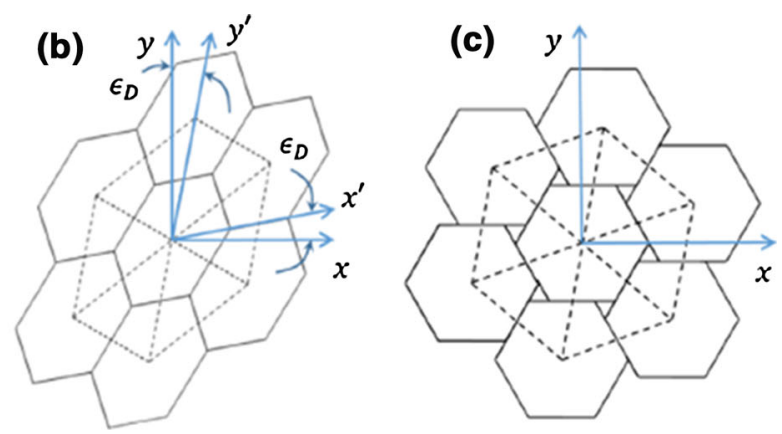


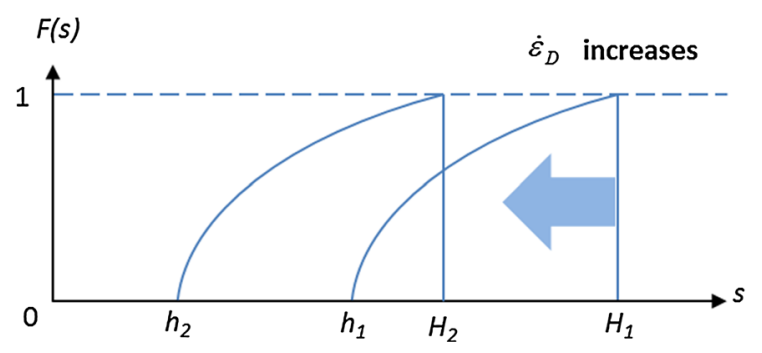

Fig. 2 Illustration of the distribution of particle size subject to increase of the effective deviatoric strain rate

$S=\int_{s=h}^{H} \frac{c_{s}}{s} \mathrm{~d} F(s)=\frac{C_{s}}{h}$

where $C_{s}=\frac{c_{s} k}{k-1} \frac{(H / h)^{k-1}-1}{(H / h)^{k}-1}$ is a dimensionless constant (Bažant and Caner 2014). For $H / h=10$ and $k=0.5, C_{s}=1.0465$.

Equation (5) adds, or integrates, the energy dissipations for different particle sizes are calculated under the assumption that for each size there is a regular array of equal-size particles. This is certainly a simplification, and it must be admitted that its error could be significant for a very broad range. In reality a large forming particle will be in contact with a small one. However, kinetically driven interface fracture creating adjacent particles of different size is hardly amenable to an analytical solution needed for the constitutive law. The error due to this simplification could be explored numerically, but is beyond the scope of this study.

\section{The criterion of dynamic fracture}

Two forms of dynamic fracture criterion were considered in (Bažant and Caner 2014; Bažant and Caner (2013)):

Either $\mathrm{d}(\Delta \mathcal{K}+S \Gamma) / \mathrm{d} S=0$

or $-\Delta \mathcal{K}=S \Gamma$

where $\Gamma=$ interface fracture energy of the comminuting particles. The former criterion, Eq. (6), is analogous to classical fracture mechanics in which the strain energy energy is being dissipated gradually at one place, the tip of a propagating crack. This criterion (Eq. 6) implies that the fracture will propagate once it is triggered. The latter one (Eq. 7) imposes an overall energy balance condition which would not guarantee incremental energy balance at the beginning of propagation. Here, however, it is hard to imagine propagation. In dynamic comminution, the fracture forms and the kinetic energy is getting dissipated simultaneously and suddenly at many places, at all the interfaces corresponding to the same point of the smearing macrocontinuum. So, the latter criterion, Eq. (7), appears more realistic. Nevertheless, either criterion leads to equations of the same form and the difference would matter only in a fully predictive model that is not calibrated by macroscale tests.

Substitution of Eqs. (3) and (5) into Eq. (7) gives the minimum particle size as

$h=\left(\frac{C_{a} \Gamma}{\rho \dot{\epsilon}_{D}^{2}}\right)^{1 / 3}$

where

$C_{a}=\frac{C_{s}}{C_{k}}=\frac{c_{s}}{c_{k}} \frac{k+2}{k-1} \frac{(H / h)^{k-1}-1}{(H / h)^{k+2}-1}$

which is a dimensionless constant; for $H / h=10$ and $k=0.5, C_{a}=0.6891$. Note that here $C_{a}$ is slightly different from its value in the first version of the theory (Bažant and Caner 2014), in which the former dynamic fracture criterion was adopted.

As seen in Eq. (8), the particle size is proportional to the $-2 / 3$ power of the effective deviatoric strain rate. A higher effective deviatoric strain rate gives a greater kinetic energy release, which leads to smaller particles, and thus to a greater interface area and more dissipation. Furthermore, substitution of Eq. (8) into Eq. (3) gives the kinetic energy drop in terms of only the effective deviatoric strain rate and the material parameters:

$-\Delta \mathcal{K}=\left(C_{0} \Gamma^{2} \rho\right)^{1 / 3} \dot{\epsilon}_{D}^{2 / 3}$

where $C_{0}=C_{k}^{3} C_{a}^{2}$ is a dimensionless constant. For $H / h=10$ and $k=0.5, C_{0}=1.6632$.

In Bažant and Caner (2013) and Bažant and Caner (2014), it was proposed that the type of behavior is decided by the dimensionless number

$B_{a}=\frac{-\Delta \mathcal{K}}{\tau_{0}^{2} /\left(2 G_{s}\right)}$

which represents the ratio of the kinetic energy density of shear to the maximum possible strain energy density, the latter being $\tau_{0}^{2} /\left(2 G_{S}\right)$ where $\tau_{0}=$ shear strength and $G_{s}=$ elastic shear modulus of the solid. Kinetic comminution must happen when $B_{a} \gg 1$ and cannot happen when $B_{a} \ll 1$. For concrete, the transition occurs at $\dot{\epsilon}_{D} \approx 1 /$ s. In Bažant and Caner (2013) and Bažant and Caner (2014), Eq. (11) could be transformed to the form: $B_{a}=G /\left(C_{g} \tau^{2}\right)\left(\Gamma^{2} \rho \dot{\epsilon}_{D}^{2}\right)^{1 / 3}$. Here this expression cannot be derived because the oversimplifying 
hypothesis from the previous works is not adopted here. However, for physical reasons, this expression is probably applicable at least as an approximation.

\section{Viscosity dissipating comminution energy in computations}

The material comminution represents an additional mechanism of the dissipation of energy. The precise way in which the comminution energy is dissipated in numerical simulations appears to make little difference for the macroscopic structural effects of impact. In simulations, the energy either can be dissipated as the work of a body force or can be incorporated into the constitutive law as an additional stress. The two ways are equivalent, in principle. An additional viscous stress is preferred here since it is easier to program and allows incorporating different types of comminution.

Since the comminution is induced by shear, the additional stress resisting the motion should be applied only to the shear stress components. The work of additional shear stresses must dissipate an energy equal to the drop of kinetic energy of strain rate field caused by comminution; i.e.

$\mathrm{d}(-\Delta \mathcal{K})=s_{i j}^{A} \mathrm{~d} \epsilon_{D i j}$

where $i, j=1,2,3, \mathrm{~d}(.$.$) denotes a small increment$ during time increment $\mathrm{d} \Delta t$. This increment is easy to understand and program if the additional stress is defined as a viscous stress;

$s_{i j}^{A}=\eta_{D} \dot{\epsilon}_{D i j}$

Then, Eqs. (10-13) give the viscosity as

$\eta_{D}=\frac{1}{2 \dot{\epsilon}_{D}^{2}} \frac{\mathrm{d}(-\Delta \mathcal{K})}{d t}=\frac{\left(C_{0} \Gamma^{2} \rho\right)^{1 / 3} \ddot{\epsilon}_{D}}{3 \dot{\epsilon}_{D}^{7 / 3}}$

It is found that, in contrast to the previous simplified formulation (Bažant and Caner 2014), the viscosity is not only related to the effective deviatoric strain rate, but is also proportional to its derivative, i.e. the 2 nd time derivative of strain. The comminution occurs only if the effective deviatoric strain rate changes, and no additional comminution occurs during the time intervals in which the rate is constant, which is a significant difference from the previous formulation (Bažant and Caner 2013; Bažant and Caner 2014).

To verify the theory, consider the following simple shear variation:

$\epsilon_{12}=\epsilon_{21}=\frac{t^{2}}{(\Delta t)^{2}} \epsilon_{\max }$ while all other strain components remain zero. Here $\epsilon_{\max }$ is the shear strain at $t=\Delta t$. According to Eq. (10), the kinetic energy drop during $t \in[0, \Delta t]$ is obtained as:

$-\Delta \mathcal{K}=\left(C_{0} \Gamma^{2} \rho\right)^{1 / 3}\left(\frac{2 \epsilon_{\max }}{\Delta t}\right)^{2 / 3}$

On the other hand, the additional stress for the simple shear case is

$s_{12}^{A}=s_{21}^{A}=\frac{\left(C_{0} \Gamma^{2} \rho\right)^{1 / 3}}{3}\left(\frac{2 t^{4} \epsilon_{\max }}{(\Delta t)^{2}}\right)^{-1 / 3}$

The work of the additional stress on the deviatoric strain can be calculated by integration over $t \in[0, \Delta t]$;

$D=2 \int_{\epsilon_{12}=0}^{\epsilon_{\max }} s_{i j}^{A} \mathrm{~d} \epsilon_{12}=\left(C_{0} \Gamma^{2} \rho\right)^{1 / 3}\left(\frac{2 \epsilon_{\max }}{\Delta t}\right)^{2 / 3}$

As is seen, the work of the additional stress calculated according to the present formulation exactly equals the kinetic energy drop induced by comminution, i.e. $D=$ $-\Delta \mathcal{K}$. So the new version of the kinetic comminution theory works correctly.

\section{Post-comminution behavior}

The comminution process (Fig. 1) is a transient process. It occurs virtually instantaneously. However, what happens if the effective deviatoric strain rate increases or decreases in the post-comminution regime, i.e., after the comminution occurred? Since, according to experiments, the particle size $s$ is not uniform but distributed between certain limits, from $h$ to $H$, it is natural to expect that the previously formed particles in practice instead of uniform size, the bigger size particles can be comminuted further into smaller particles when the strain rate increases, while the relative size distribution still holds true, as shown in Fig. 2. Thus, during a strain rate increase, further interfaces are created to dissipate more kinetic energy. The additional stress and viscosity that dissipate this energy can be obtained from Eqs. (12-14).

The effective deviatoric strain rate as a function of time can in practice evolve in a complicated manner, as shown by the response in Fig. 3 separated into stages I, II, III and IV. The comminution occurs at the beginning of stage I and Eqs. (12-14) apply in that stage. In stage II, the effective deviatoric strain rate decreases, and so the additional stress and viscosity are zero, because the strain rate is not big enough to create smaller particles. 


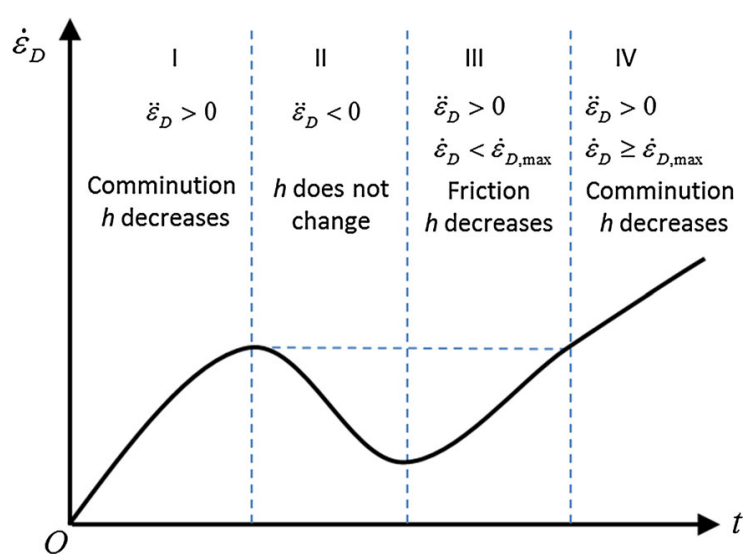

Fig. 3 Various situations of the strain rate in practice

In stage III, though, it is reasonable to assume that the small comminuted particles cluster together into bigger particle groups and release kinetic energy due to the increases of effective deviatoric strain rate. The kinetic energy released, which can be calculated by Eqs. (1214) as well, may be dissipated by the friction between particles. Further comminution must occur in stage IV as the effective deviatoric strain rate exceeds its previous maximum, and the particles are comminuted into still smaller particles. Therefore, the viscosity and additional stress in Eqs. (12-14) should be applied only when $\dot{\epsilon}_{D}>0$ and $\ddot{\epsilon}_{D}>0$. This behavior can be implemented into any existing constitutive law for the effect of comminution.

\section{Application of microplane model M7 and quasi-static rate effects}

Microplane model M7 (Caner and Bažant 2012) was chosen since it is at present the only continuum damage model that can fit all of the 22 existing types of laboratory tests uni-, bi-, and tri-axial behavior under various loading and unloading histories with post-peak softening. Since the fracture process zone size in concrete is typically about $0.3 \mathrm{~m}$, damage localization and nonlocal concepts are not very important in representing the laboratory tests. M7 was calibrated to fit the test data for concrete WES-5000 used in the tests of projectile impact. This concrete had compression strength $48 \mathrm{MPa}$, Young's modulus $E=25 \mathrm{GPa}$, and Poisson's ratio $v=0.18$. The optimum values of $\mathrm{M} 7$ parameters, achieving very good fits of quasi-static uni-, bi- and tri-axial tests, were $k_{1}=11 \times 10^{-5}, k_{2}=110, k_{3}=$ $30, k_{4}=100$ and $k_{5}=10^{-4}$ (Caner and Bažant 2012; Caner and Bažant (2012)).

The M7 includes the quasi-static rate effects, which consist of a rate-dependent crack opening and growth controlled by the activation energy of bond breakage, and of viscoelasticity of the material between the cracks. The quasi-static rate effects suffice to capture the rate-dependent behavior up the the strain rate of about $1 / \mathrm{s}$, but not beyond. In theory, they should extend into very high strain rates, even in excess of $10^{4} / \mathrm{s}$, though alone they are insufficient to fit test data.

In the zones farther away from the penetrating projectile, the strain rate is not extremely high. In these zones, only the quasi-static rate effects play a role, while the kinetic comminution effect discussed here does not. To capture the quasi-static rate effect due to cohesive crack opening controlled by the activation energy theory of bond ruptures, the M7 stress-strain boundaries on the microplane level (i.e., the straindependent strength limits for microplane stress and strain vectors) are scaled vertically in proportion to the function $F(\epsilon)\left[1+C_{r 2} \ln \left(2 \dot{\epsilon} / C_{r 1}\right)\right]$. Here, $F(\epsilon)$ is the boundary at negligible strain rate, $\dot{\epsilon}$ is an invariant of the strain tensor, $C_{r 1}=4 \times 10^{-6}$ and $C_{r 2}=0.022$. The effect of viscoelasticity between the cracks is taken into account by replacing the elastic constants with a creep operator, for which, in the case of impact modeling, a single spring-dashpot Maxwell rheologic model suffices (Bažant et al. 2000; Caner and Bažant 2000; Bažant and Caner 2014; Caner and Bažant (2014)). These quasi-static rate effects can capture the apparent strength increase in unconfined compression tests at rates only up to about $1 / \mathrm{s}$ (which is nevertheless sufficient for the effects of earthquake or remote explosions).

\section{Projectiles penetrating and perforating concrete walls}

Although the new microplane model M7 (Caner and Bažant 2012; Caner and Bažant (2012)) for concrete can provide excellent fits of the experiment data from diverse types of uniaxial, biaxial and triaxial tests, its application to projectile impact severely overestimated the exit velocities of projectiles perforating concrete walls and the depths of penetration of concrete blocks. The previous enhancement of M7 with 
simplified calculation at additional viscosity led to major improvement (Bažant and Caner 2013; Caner and Bažant (2014)), but the agreement with data was imperfect. Here, we demonstrate that the present theory of kinetic comminution leads to virtually perfect agreement.

The tests of projectile perforation, performed at the Geotechnical and Structure Laboratory of the US. Army Engineer Research and Development center (ERDC), Vicksburg (Cargile 1999; Frank et al. (2012)), used circular slabs of four thicknesses, 127, 216, 254 and $280 \mathrm{~mm}$, made of the concrete WES-5000, whose standard compression strength was $48 \mathrm{MPa}$. The slabs were cast in steel culvert pipes of diameter $1.52 \mathrm{~m}$, sufficient to approximate the response for a semi-infinite radius (in spite of that, non-reflecting finite elements producing no backward wave were used at the boundary). The projectiles, which were hollow and made of steel, had an ogival-nose (caliber radius head 3.0, length/diameter ratio 7.0, and diameter $50.8 \mathrm{~mm}$ ) and weight of $2.3 \mathrm{~kg}$. They were considered as rigid since no obvious damage, melt or deformation was observed after the test. The projectiles impacted the concrete slabs with the velocity of $310 \mathrm{~m} / \mathrm{s}$ at the angle of $90^{\circ}$. The heat generation was neglected based on simple estimates. The perforation tests were carried out two or three times for each thickness of the slab.

Figure 4 shows that the exit velocities of the projectiles were grossly underestimated, provided that all the types of artificial damping unsupported by mechanics and experiments, which are typically embedded in the commercial finite element codes, were eliminated. The additional viscosity formulated here provides a major improvement, even better than the simplified additional viscosity in Bažant and Caner (2013) and Bažant and Caner (2014). The kinetic comminution viscosity was based on $H / h=10, k=0.5$. There is only one unknown parameter for the present kinetic comminution model, namely $\alpha=C_{0} \Gamma^{2} \rho$. It can be calibrated by fitting only one data point in Fig. 4, which led to the value $\alpha=11034 \mathrm{~N}^{2} \mathrm{Kg} / \mathrm{m}^{5}\left(C_{0}=1.66, \Gamma=1.7\right.$ $\left.\mathrm{N} / \mathrm{m}, \rho=2300 \mathrm{Kg} / \mathrm{m}^{3}\right)$. The remaining points are then predicted. As can be seen, the predictions of exit velocities are excellent, for all the data points. The distributed stress with comminution is usually higher than without, since the additional viscous stress is added to implement the effect of comminution.

For the penetration test (Cargile 1999), the CSPC concrete block was big enough to be treated as semi-

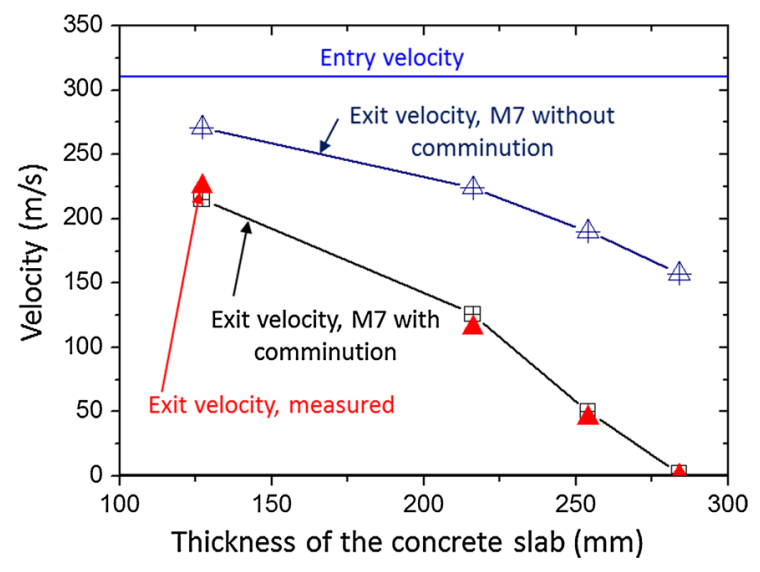

Fig. 4 Comparison of the exit velocity predicted by M7 with and without implementation of comminution effect and the experiment data for projectile perforation onto the concrete wall with thicknesses of 127, 216, 254 and $280 \mathrm{~mm}$

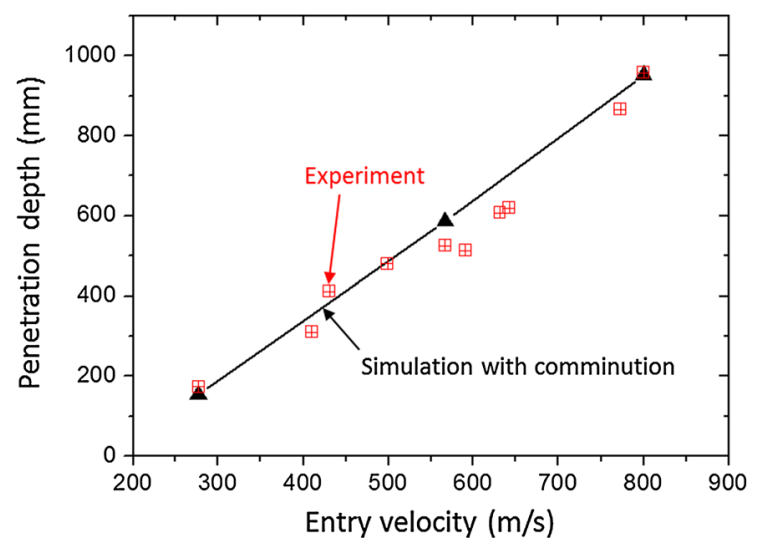

Fig. 5 Comparison of the penetration depth predicted by M7 with implementation of comminution effect and the experiment data. The entry velocity of the projectile ranges from 277 to $800 \mathrm{~m} / \mathrm{s}$

infinite. The projectiles, made of steel, were hollow, had an ogive nose, length $242.4 \mathrm{~mm}$, diameter $25.9 \mathrm{~mm}$ and weight $0.9 \mathrm{~kg}$, and are here considered as rigid since they were found undeformed after the test. The entry velocities ranged from 277 to $800 \mathrm{~m} / \mathrm{s}$. The penetration depths measured are shown in Fig. 5. The blocks consisted of the CSPC concrete, which was rather similar to the WES-5000 concrete. Therefore, the parameters for WES-5000 are applied here. Again, only one free parameter, $\alpha=C_{0} \Gamma^{2} \rho$, was available to fit the data, and the value $\alpha=61090 \mathrm{~N}^{2} \mathrm{Kg} / \mathrm{m}^{5}$, along with $C_{0}=1.66, \Gamma=4.0 \mathrm{~N} / \mathrm{m}, \rho=2300 \mathrm{Kg} / \mathrm{m}^{3}$, provided the optimum fit. As shown in Fig. 5, three typical cases 
with entry velocities of 277,567 and $800 \mathrm{~m} / \mathrm{s}$ were simulated. The thicknesses of the concrete blocks were 760,1070 and $1830 \mathrm{~mm}$, respectively, according to the experiment. The simulations agree with the test data very well.

For comparison, the predictions without the consideration of the comminution were also done. The penetration depths were 531 and $1606 \mathrm{~mm}$ for the first and the third cases, respectively. However, for the second case, the exit velocity calculated without comminution was $153.7 \mathrm{~m} / \mathrm{s}$, which is far from the measured value.

Besides the exit velocity and penetration depth, the shape of the entry and exit craters is another result that should be compared with experiments. It depends on various factors, such as the criterion for element deletion, which must be used to avoid excessive element distortion. Here, the elements with principal strain below $-20 \%$ or above $100 \%$ are deleted. The craters obtained by the present simulations do not fit the experiments well; they are much too narrow. This problem will be studied further.

\section{Conclusions and final comments}

1. The recent idea (Bažant and Caner 2014) to model the dynamic overstress in projectile penetration by means of comminution driven by local kinetic of shear strain rate is here extended and refined by deriving from the energy balance a more accurate expression for the additional apparent viscosity that generates the overstress.

2. The basic hypothesis is that the interface fracture that comminutes the material into small particles occurs instantly, as soon as energy equivalent of the local kinetic energy of forming particles becomes equal to the fracture energy.

3. In contrast to the previous simplified formulation, the additional apparent viscosity, which supplements the quasi-static rate effects, involves a different exponent of the effective deviatoric strain rate and depends not only on the effective deviatoric strain rate but also on its time derivative, i.e., on the second derivative of the effective deviatoric strain.

4. Because of the second derivative, no further kinetic comminution is under way if the effective deviatoric strain rate remains constant.
5. The post-comminution behavior, including subsequent further comminution and clustering into bigger particle groups to release the kinetic energy that dissipated by friction between the groups, is discussed and incorporated into the present improved theory.

6. When the present model is implemented in a commercial finite element code, the artificial damping of all types, normally embedded in these codes, must be eliminated since it is non-physical, nonpredictive.

7. In combination with microplane model M7 which also includes the quasi-static rate effects, excellent predictions are obtained for the exit velocities of projectiles penetrating walls of different thicknesses and for the depths or penetration of projectiles of different velocities into concrete blocks.

8. As a final comment, a rational theory of high rate shear comminution might have an important use in the technology of hydraulic fracturing of gas or oil shale. Oil companies are currently investigating powerful explosions in the horizontal welbore, which would have to be done by electro-hydraulic pulsed arc since enough chemical explosive cannot be packed into the narrow wellbore. Although, due to rapid front attenuation in 3D shock front expansion, only a part of the frac stage could be comminuted, the fragmentation near the wellbore could stimulate the flow through the cracks, mitigate proppant screening and enhance permeability.

Acknowledgments Support during the initial phase of research under grant W911NF-09-1-0043/P00003 from the U.S. Army Research Office, Durham, to Northwestern University, is gratefully acknowledged. So is an additional support obtained from the ADD, Korea, through a Daejeon University grant to Northwestern University. Thanks are due to Ferhun Caner, Visiting Scholar on leave from UPC, Barcelona, for making available his coding of M7 and the meshes for slab perforations.

\section{References}

Adley MD, Frank AO, Danielson KT (2012) The high-rate brittle microplane concrete model: Part I: bounding curves and quasi-static fit to material property data. Comput Concr 9:293-310

Bažant ZP, Caner FC, Carol I, Adley MD, Akers SA (2000) Microplane model M4 for concrete. I: formulation with work-conjugate deviatoric stress. J Eng Mech 126:944-953

Bažant ZP, Caner FC (2013) Comminution of solids caused by kinetic energy of high shear strain rate, with implications for impact, shock, and shale fracturing. Proc Natl Acad Sci 110:19291-19294 
Bažant ZP, Caner FC (2014) Impact comminution of solids due to local kinetic energy of high shear strain rate: I. Continuum theory and turbulence analogy. J Mech Phys Solids 64:223235

Bažant ZP, Su Y (2015) Impact comminution of solids due to progressive crack growth driven by kinetic energy of highrate shear. ASME J Appl Mech 82:031007-1-031007-5

Cadoni E, Labibes K, Albertini C, Berra M, Giangrasso M (2001) Strain-rate effect on the tensile behaviour of concrete at different relative humidity levels. Mater struct 34:21-26

Camacho G, Ortiz M (1996) Computational modelling of impact damage in brittle materials. Int J Solids Struct 33:28992938

Caner FC, Bažant ZP (2000) Microplane model M4 for concrete. II: algorithm and calibration. J Eng Mech 126:954-961

Caner FC, Bažant ZP (2012a) Microplane model M7 for plain concrete. I: formulation. J Eng Mech 139:1714-1723

Caner FC, Bažant ZP (2012b) Microplane model M7 for plain concrete. II: calibration and verification. J Eng Mech 139:1724-1735

Caner FC, Bažant ZP (2014) Impact comminution of solids due to local kinetic energy of high shear strain rate: II. Microplane model and verification. J Mech Phys Solids 64:236-248

Cargile JD (1999) Development of a constitutive model for numerical simulation of projectile penetration into brittle geomaterials. DTIC Document

Charles RJ (1957) Energy-size reduction relationships in comminution. Trans Am Inst Min Metall Eng 208:80-88

Deshpande V, Evans A (2008) Inelastic deformation and energy dissipation in ceramics: a mechanism-based constitutive model. J Mech Phys Solids 56:3077-3100

Doyoyo M (2002) A theory of the densification-induced fragmentation in glasses and ceramics under dynamic compression. Int J Solids Struct 39:1833-1843

Ferri E, Deshpande V, Evans A (2010) The dynamic strength of a representative double layer prismatic core: a combined experimental, numerical, and analytical assessment. J Appl Mech 77:061011

Forquin P, Gary G, Gatuingt F (2008) A testing technique for concrete under confinement at high rates of strain. Int $\mathrm{J}$ Impact Eng 35:425-446

Frank AO, Adley MD, Danielson KT, McDevitt HS Jr (2012) The high-rate brittle microplane concrete model: Part II: application to projectile perforation of concrete slabs. Comput Concr 9:311-325

Freund LB (1990) Dynamic fracture mechanics. Cambridge University Press, Cambridge

Gailly BA, Espinosa HD (2002) Modelling of failure mode transition in ballistic penetration with a continuum model describing microcracking and flow of pulverized media. Int J Numer Methods Eng 54:365-398
Gatuingt F, Pijaudier-Cabot G (2002) Coupled damage and plasticity modelling in transient dynamic analysis of concrete. Int J Numer Anal Methods Geomech 26:1-24

Grady D, Kipp M (1979) The micromechanics of impact fracture of rock. Int J Rock Mech Min Sci Geomech Abstr 16:293302

Grady D (1982) Local inertial effects in dynamic fragmentation. J Appl Phys 53:322-325

Grady DE (1990) Particle size statistics in dynamic fragmentation. J Appl Phys 68:6099-6105

Grady D, Kipp M (1995) Experimental measurement of dynamic failure and fragmentation properties of metals. Int J Solids Struct 32:2779-2791

Grady D (1998) Shock-wave compression of brittle solids. Mech Mater 29:181-203

Hemmert DJ, Smirnov VI, Awal R, Lati S, Shetty A (2010) Pulsed power generated shockwaves in liquids from exploding wires and foils for industrial applications. In: Proceedings of the 16th International Symposium on High Current Electronics, Tomsk, Russia, pp 537-540

Kožar I, Ožbolt J (2010) Some aspects of load-rate sensitivity in visco-elastic microplane material model. izlazi u samo elektronikom izdanju: NE

Maurel O, Reess T, Matallah M, De Ferron A, Chen W, La Borderie C, Pijaudier-Cabot G, Jacques A, Rey-Bethbeder F (2010) Electrohydraulic shock wave generation as a means to increase intrinsic permeability of mortar. Cem Concr Res 40:1631-1638

Mescall J, Weiss V (1984) Materials behavior under high stress and ultrahigh loading rates Part II, In: Proceeding of 29th Sagamore Army Conference. Army Materials and Mechanics Center, Watertown, MA

Mott N (1947) Fragmentation of shell cases. Proce R Soc Lond Ser A Math Phys Sci 189:300-308

Ouchterlony F (2005) The Swebrec function: linking fragmentation by blasting and crushing. Min Technol 114:29-44

Ožbolt J, Sharma A, Reinhardt H-W (2011) Dynamic fracture of concrete compact tension specimen. Int J Solids Struct 48:1534-1543

Schuhmann R Jr (1940) Principles of comminution, I-size distribution and surface calculations. AIME Technical Publication, Los Angeles 1189

Shih C, Nesterenko V, Meyers M (1998) High-strain-rate deformation and comminution of silicon carbide. J Appl Phys 83:4660-4671

Wei Z, Evans A, Deshpande V (2009) The influence of material properties and confinement on the dynamic penetration of alumina by hard spheres. J Appl Mech 76:051305 